\title{
Hard X-ray emission from Eta Carinae \& other colliding-wind binaries
}

\author{
J.-C. Leyder ${ }^{* \dagger a b}$, R. Walter ${ }^{b}$ and G. Rauw ${ }^{\ddagger} a$ \\ ${ }^{a}$ Institut d'Astrophysique et de Géophysique, Université de Liège \\ ${ }^{b}$ INTEGRAL Science Data Centre, Observatoire de Genève \\ E-mail: leydereastro.ulg.ac.be
}

If relativistic particle acceleration takes place in colliding-wind binaries, hard X-rays and $\gamma$-rays are expected through inverse Compton scattering of the copious UV radiation, but they had never been unambiguously detected.

To detect this emission, observations performed with INTEGRAL were carefully analysed, taking advantage of the high spatial resolution of this satellite with respect to previous $\gamma$-ray missions. In particular, deep hard X-ray images of the region of Eta Carinae were constructed in several energy bands, leading to the very first detection of this source at energies up to $100 \mathrm{keV}$. The hard X-ray emission previously detected by BeppoSax around Eta Car originates from at least 3 different point sources. The emission of Eta Car itself can be isolated for the first time, and its spectrum unambiguously analyzed.

The X-ray emission of Eta Car in the $22-100 \mathrm{keV}$ energy range is very hard (with a photon index $\Gamma$ around 1), and its luminosity is $7 \times 10^{33} \mathrm{erg} / \mathrm{s}$. The observed emission is in agreement with the predictions of inverse Compton models, and corresponds to about $0.1 \%$ of the energy available in the wind collision.

A systematic search for hard X-ray emission from other colliding-wind binaries and from massive stars is also being performed using the INTEGRAL data archive, and the first results are presented.

7th INTEGRAL Workshop

September 8-11 2008

Copenhagen, Denmark

\footnotetext{
*Speaker.

$\dagger$ FNRS Research Fellow.

${ }^{\ddagger}$ FNRS Research Associate.
} 


\section{Introduction}

The rationales behind the search for hard X-ray and $\gamma$-ray emission from colliding-wind binaries (CWBs) with INTEGRAL are presented in Section 1. Then, the observations of Eta Carinae are described in Section 2. Finally, the hard X-ray emission expected from other CWBs is briefly discussed in Section 3, and future prospects are offered in Section 4.

\section{High-energy emission from colliding-wind binaries}

A colliding-wind binary (CWB) is a binary system consisting of two massive stars, each having a strong stellar wind. These two winds collide, leading to the formation of a hydrodynamical shock (see Figure 1, taken from the website of the High-Energy Astrophysics group from Liège), where particle acceleration can take place. In particular, electrons can be accelerated up to relativistic energies by the first-order Fermi mechanism (or "diffusive shock acceleration"). This mechanism is based on the presence of a hydrodynamical shock moving at speed $v$ : each time the particles cross the shock, energy is transferred to the particles. The energy gain is proportional to the first order in $v / c$ (hence the name) $[1,2,3]$. This is a variant of the original scenario suggested by Fermi in 1949 [4], where the particles collide with a "magnetic mirror" moving at speed $v$ : they can gain or loose energy, although there is a gain on average. The net energy gain is proportional to $(v / c)^{2}$, and the process is nowadays known as the second-order Fermi mechanism.

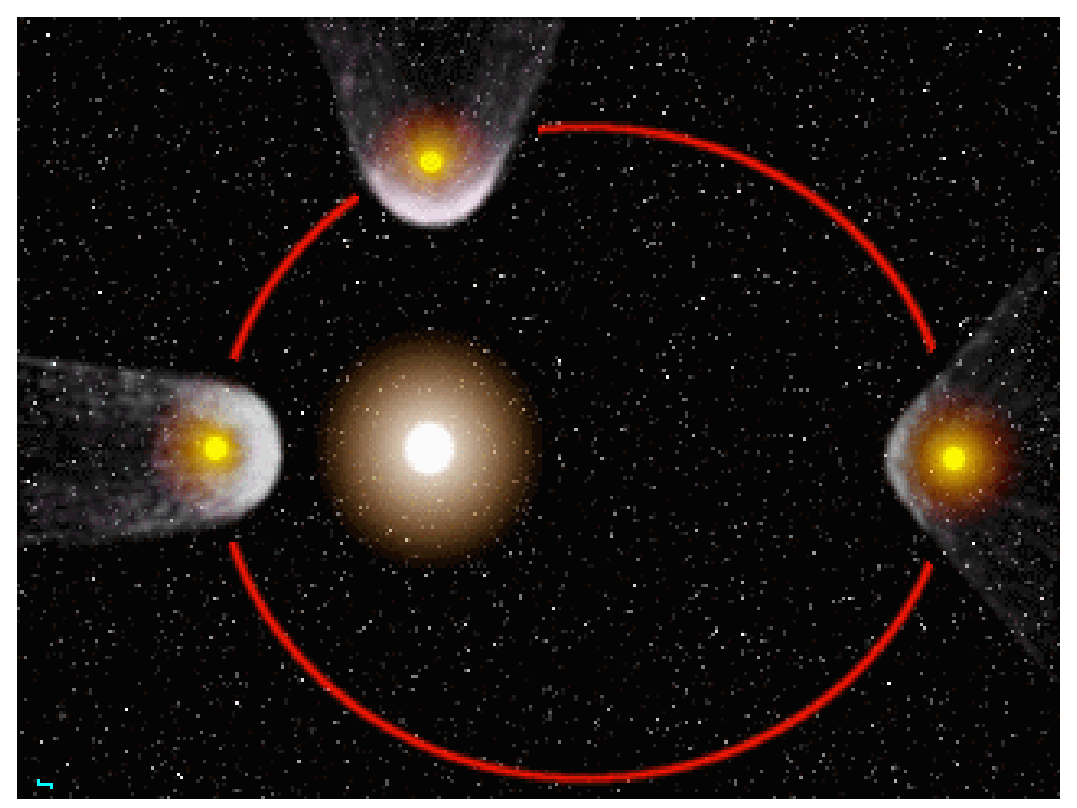

Figure 1: Schematic illustration of a colliding-wind binary (CWB) system, represented at three different moments along the orbital period. The shape of the shock depends on the respective strengths of the two winds, and varies with the orbital phase.

Moreover, massive stars are early-type stars, and as such, they exhibit a strong UV radiation field. The combined presence of the relativistic electrons and of the UV radiation field implies that the inverse Compton mechanism can take place, thus explaining why a hard X-ray and $\gamma$-ray emission is expected from CWBs $[1,2,3]$. 


\section{Hard X-ray emission from Eta Carinae}

\subsection{A few words on Eta Carinae}

Eta Carinae is mostly known for its "Great eruption" in 1843 [5]. Its mass-loss rate is derived to be $10^{-4}-10^{-3} \mathrm{M}_{\odot} /$ year. It is known to be a binary system, as an orbital period of 5.5 years has been detected using optical [6], infrared [7], and X-ray observations [8]; a high eccentricity $(e \simeq 0.9)$ has been inferred [9]. It harbours a Luminous Blue Variable and a less extreme (O- or WR-type) star [10]. Eta Car has also been classified as a colliding-wind binary, based on its X-ray spectrum [8].

\subsection{Previous BeppoSAX observations of Eta Car}

There are four BeppoSAX observations of the Carina region, performed with the non-imaging PDS instrument [11]. Three of the observations revealed a high-energy excess (in the 13-20 keV energy range), at the orbital phases ${ }^{1} \Phi=0.83,1.37$, and 1.46. No excess was seen at $\Phi=1.05$, although this needs confirmation due to possible source confusion (see Section 2.3). Moreover, one of the BeppoSAX observations showed a high-energy tail up to $50 \mathrm{keV}$ (in June 2000, corresponding to $\Phi=1.46$ ).

\subsection{Current INTEGRAL observations of Eta Car}

Using INTEGRAL observations, images of the Carina region were built in different energy bands. Eta Carinae is detected in the $22-100 \mathrm{keV}$ image (see Figure 2), with a significance larger than 7 , and a luminosity of $7 \times 10^{33} \mathrm{erg} / \mathrm{s}$ [12]. In addition, two other faint sources are detected within the BeppoSAX/PDS field of view : the anomalous X-ray pulsar 1E 1048.1-5937, and a new source (IGR J10447-6027).

A spectrum of Eta Carinae could also be extracted, showing that the source is detected up to $100 \mathrm{keV}$ (see Figure 3). Fitting this spectrum with an absorbed and optically thin thermal model (wabs $\star$ mekal; temperature $k T=5.1 \mathrm{keV}$, column density $N_{H}=4.3 \times 10^{22} \mathrm{~cm}^{-2}$, in close agreement with the values of $k T=5.1 \mathrm{keV}$ and of $N_{H}=3.8 \times 10^{22} \mathrm{~cm}^{-2}$ found by [11]) is not possible without adding another component to account for the high-energy tail (powerlaw; photon in$\operatorname{dex} \Gamma=1 \pm 0.4)$.

This is thus the very first detection of high-energy non-thermal emission from a colliding-wind binary. It is very likely due to inverse Compton scattering of UV or optical photons by high-energy electrons accelerated in the wind collision zone [13].

The total power in stellar wind interactions can be evaluated as $L=\frac{1}{2} \Theta \dot{M} v^{2}$ (where $\Theta$ is a geometrical factor; [14]), leading to a value of $L_{1}+L_{2} \simeq 10^{37} \mathrm{erg} / \mathrm{s}$ using the parameters adequate for Eta Car. This means that the luminosity observed for Eta Car $\left(\simeq 10^{34} \mathrm{erg} / \mathrm{s}\right)$ corresponds to only $0.1 \%$ of the total power involved in stellar wind interactions.

The current set of INTEGRAL data covers mostly 3 major periods of observations, summarized in Table 1.

\footnotetext{
${ }^{1} \Phi=1$ corresponds to the (spectroscopic) 1998 minimum. As the orbital period is quite long (5.5 years), phases greater than 1 are used to refer to the next orbital cycle; therefore, $\Phi=2$ corresponds to the 2003 minimum, and $\Phi=3$ corresponds to the 2009 minimum.
} 


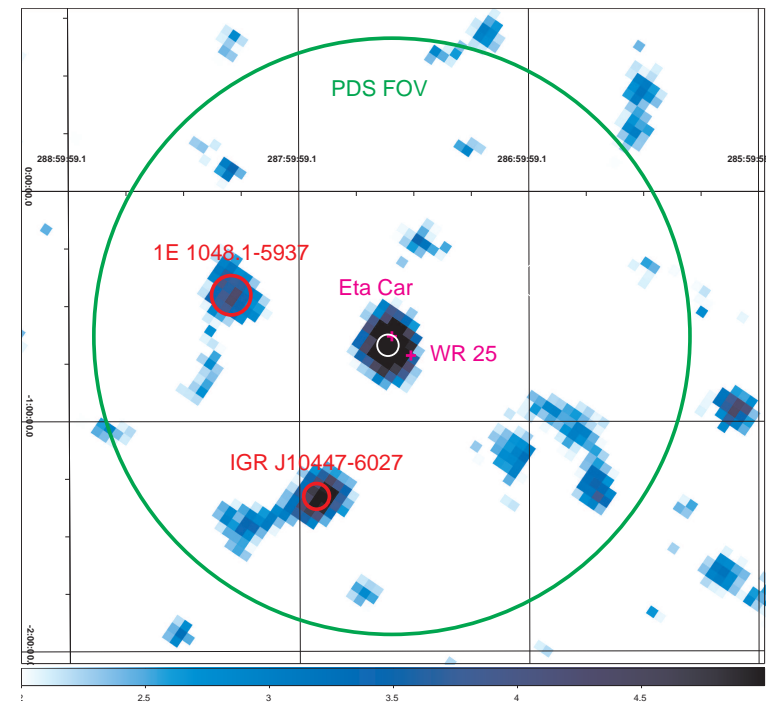

Figure 2: INTEGRAL image of the Carina region (22-100 keV; effective exposure time of $1.3 \mathrm{Ms}$ ). Three sources are detected within the BeppoSAX/PDS field of view : Eta Car, 1E 1048.1-5937 and IGR J104476027. (Note that WR 25 has been ruled out as a possible counter-part of Eta Car, as it consistently remains outside of the INTEGRAL error circle for all energy bands studied.)

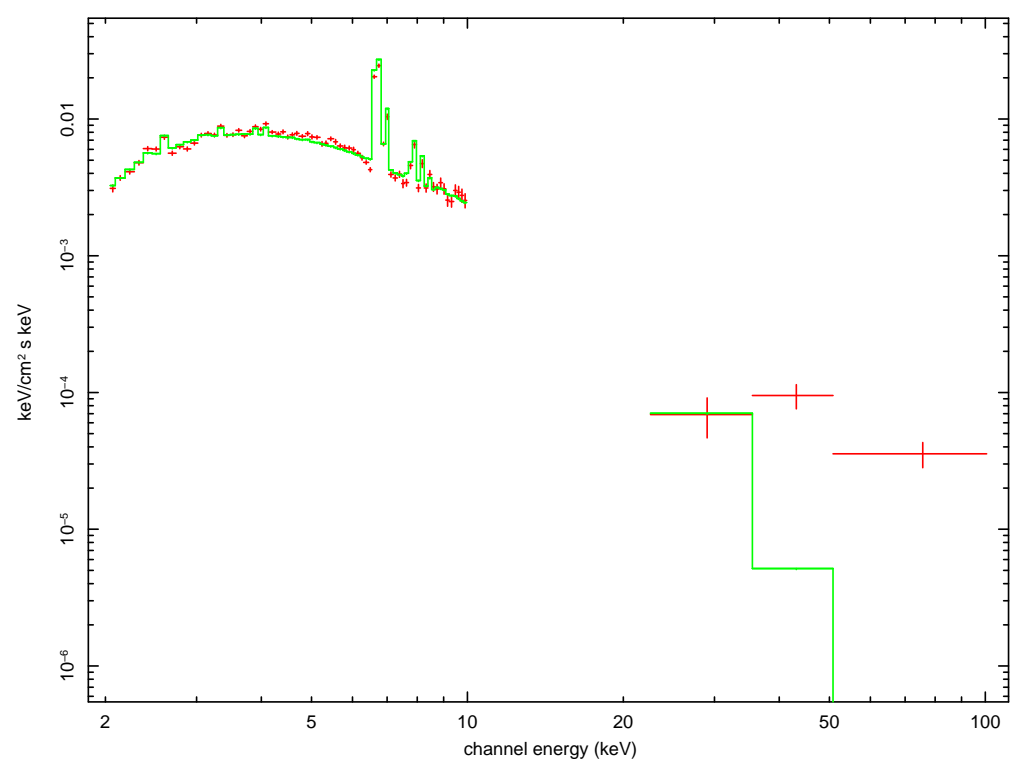

Figure 3: Spectrum of Eta Car, combining BeppoSAX and INTEGRAL data (in red). The green fit is an absorbed and optically thin thermal model (wabs $\star$ mekal; temperature $k T=5.1 \mathrm{keV}$ ) that does not reproduce the high-energy tail, hence the need for an additional component. 


\begin{tabular}{ccccc}
\hline \hline Period & Rev. & Phase $\Phi$ of $\eta$ Car & Exposure time & Significance \\
\hline 1 & $76-88$ & $1.99-2.01$ & $122 \mathrm{ks}$ & - \\
2 & $192-209$ & $2.16-2.19$ & $717 \mathrm{ks}$ & 6.2 \\
3 & $322-330$ & $2.35-2.37$ & $180 \mathrm{ks}$ & 3.3 \\
\hline All data & & & $1113 \mathrm{ks}$ & 7.3 \\
\hline
\end{tabular}

Table 1: The main INTEGRAL public data available for $\eta$ Car cover mostly three epochs. The corresponding revolution numbers, orbital phase, effective exposure time and significance of the detection of Eta Car (in the 22-100 keV energy range) are given for each period, as well as for the whole data set.

The X-ray lightcurve of Eta Car [15] is shown in Figure 4. Its general shape is well explained by a CWB scenario : during most of the orbit, the wind collision leads to the emission of a (more or less) constant X-ray flux. When approaching periastron (i.e. at $\Phi=1$ ), the emitted X-ray flux increases, as the two stars are getting closer to each other. Then the secondary moves deeper into the huge wind of the primary, hence the increase of the column density explains the drop observed in the X-ray flux. Finally, as the two components get further away from periastron, the flux comes back to its previous value. However, although Eta Car has been widely observed during the previous periastron passages in 1998 and 2003, and although the column density has been found to increase sharply, the extremely high level necessary to explain the low X-ray flux has never been observed. Therefore, it is believed that, in addition to the change of column density, a decrease of the plasma emission measure is also needed (such as the one that would result from a modification of the shock, or even its disappearance) to fully reproduce the shape of the X-ray lightcurve. This question can be probed with INTEGRAL observations taken at periastron, as a simple increase of the column density would not affect the flux observed in hard X-rays, while a change of the shock geometry and/or its disappearance would clearly be noticed. During the next periastron passage in January 2009 (at $\Phi=3$ ), 1 Ms of INTEGRAL observations will be dedicated to the Carina region, and will very likely lead to new constraints on the behaviour of the shock at periastron.

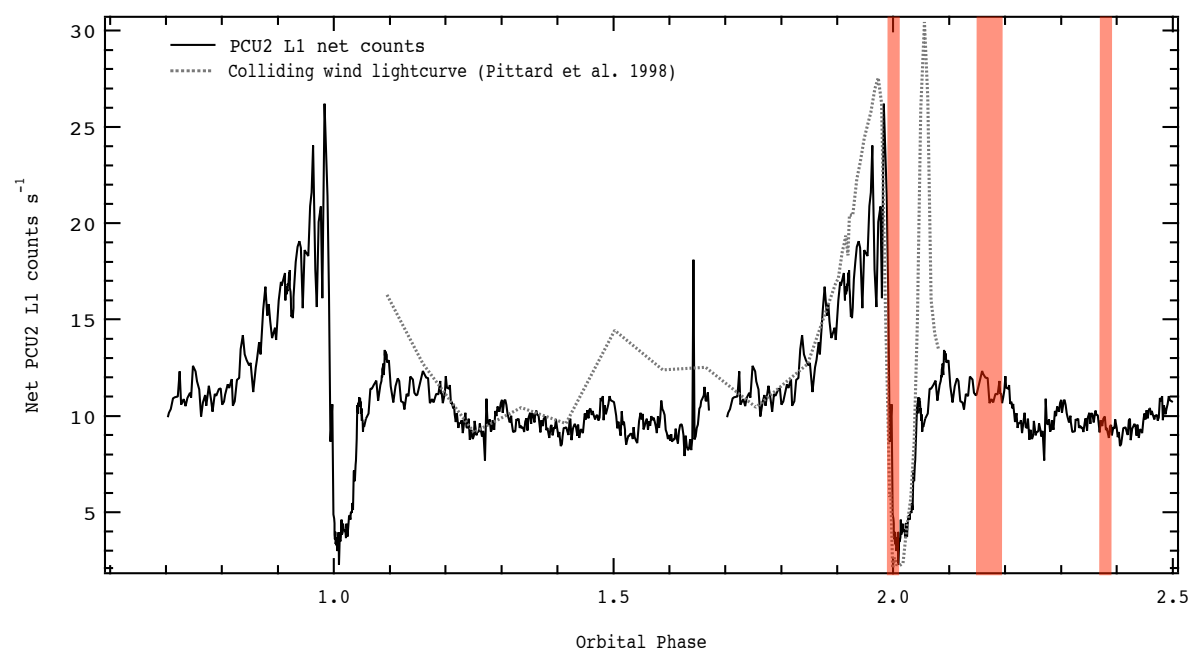

Figure 4: X-ray lightcurve of Eta Car [15], with a CWB simulation (dotted line). The vertical red bands correspond to the three major periods of observations with INTEGRAL. 


\section{INTEGRAL observations of other colliding-wind binaries}

Following the detection of Eta Car, a search for hard X-ray emission from other collidingwind binaries was initiated; a few candidates will be investigated. The most promising candidate to date is the Wolf-Rayet star WR 115. New optical observations confirmed its spectral type to be WN6, and provide evidence for the presence of an OB companion (as suggested by the presence of absorption lines). The XMM-Newton observation that was recently performed led to the detection of the WR star (see Figure 5), which is likely to indicate that WR 115 is a binary : indeed, no single WR star from the WN subtype has been unambiguously detected so far in the X-rays [16]. However, another strong X-ray source, previously unknown, lays inside the error box of the INTEGRAL detection, and is likely to be the counter-part of the hard X-ray emission. Thus, no conclusive proof of the hard X-ray emission from a CWB other than Eta Car has been observed to date.

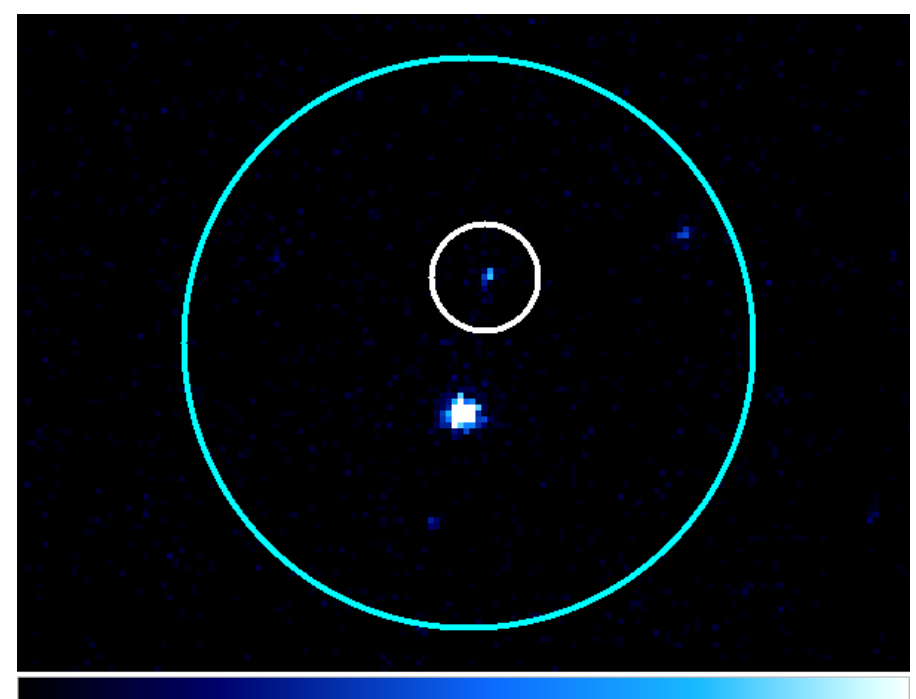

Figure 5: XMM image of WR 115 (shown in the white circle). The blue circle indicates the ISGRI error box.

\section{Future prospects}

A systematic search for the hard X-ray emission from Wolf-Rayet stars, non-thermal radio emitting early-type stars, and O-type stars (with a magnitude $V<8$ ) is currently being performed.

The variability of the hard X-ray flux observed from Eta Car will be tested by INTEGRAL observations in early 2009, thus allowing to test the physical models describing the behaviour of this CWB at periastron.

\section{Acknowledgments}

This research has made use of the SIMBAD database (CDS), of public data from INTEGRAL (ESA) \& BeppoSAX (ASI). J.-C.L. and G.R. acknowledge support through the XMM-INTEGRAL PRODEX project. 


\section{References}

[1] A. R. Bell, The acceleration of cosmic rays in shock fronts. I, MNRAS 182 (Jan., 1978) 147-156.

[2] A. R. Bell, The acceleration of cosmic rays in shock fronts. II, MNRAS 182 (Feb., 1978) 443-455.

[3] J. M. Pittard and S. M. Dougherty, Radio, X-ray, and $\gamma$-ray emission models of the colliding-wind binary WR140, MNRAS 372 (Oct., 2006) 801-826, [arXiv : astro-ph/ 0603787 ].

[4] E. Fermi, On the Origin of the Cosmic Radiation, Physical Review 75 (Apr., 1949) 1169-1174.

[5] R. Viotti, A Historical Overview of the Eta Carinae Problem, in Revista Mexicana de Astronomia y Astrofisica Conference Series (V. Niemela, N. Morrell, and A. Feinstein, eds.), vol. 2 of Revista Mexicana de Astronomia y Astrofisica Conference Series, pp. 1-+, June, 1995.

[6] A. Damineli, A. Kaufer, B. Wolf, O. Stahl, D. F. Lopes, and F. X. de Araújo, $\eta$ Carinae: Binarity Confirmed, ApJ 528 (Jan., 2000) L101-L104, [arXiv: astro-ph/9912387].

[7] P. A. Whitelock, M. W. Feast, F. Marang, and E. Breedt, The 2003 shell event in $\eta$ Carinae, MNRAS 352 (Aug., 2004) 447-456, [arXiv: astro-ph/ 0404513$].$

[8] M. F. Corcoran, X-Ray Monitoring of $\eta$ Carinae: Variations on a Theme, AJ 129 (Apr., 2005) 2018-2025.

[9] M. F. Corcoran, J. H. Swank, R. Petre, K. Ishibashi, K. Davidson, L. Townsley, R. Smith, S. White, R. Viotti, and A. Damineli, The Chandra HETGS X-Ray Grating Spectrum of $\eta$ Carinae, ApJ 562 (Dec., 2001) 1031-1037, [arXiv:astro-ph/0105335].

[10] R. C. Iping, G. Sonneborn, T. R. Gull, D. L. Massa, and D. J. Hillier, Detection of a Hot Binary Companion of $\eta$ Carinae, ApJ 633 (Nov., 2005) L37-L40, [arXiv: astro-ph/ 0510581 ].

[11] R. F. Viotti, L. A. Antonelli, C. Rossi, and S. Rebecchi, The high energy X-ray tail of $\eta$ Car revealed by BeppoSAX, A\&A 420 (June, 2004) 527-532, [arXiv: astro-ph/ 0402329 ].

[12] J.-C. Leyder, R. Walter, and G. Rauw, Hard X-ray emission from $\eta$ Carinae, A\&A 477 (Jan., 2008) L29-L32, [0712.1491].

[13] P. Benaglia and G. E. Romero, Gamma-ray emission from Wolf-Rayet binaries, A\&A 399 (Mar., 2003) 1121-1134, [arXiv:astro-ph/0205375].

[14] J. M. Pittard and I. R. Stevens, The dominant X-ray wind in massive star binaries, A\&A 388 (June, 2002) L20-L23, [arXiv:astro-ph/0204236].

[15] J. M. Pittard and M. F. Corcoran, In hot pursuit of the hidden companion of eta Carinae: An X-ray determination of the wind parameters, A\&A 383 (Feb., 2002) 636-647, [arXiv:astro-ph/0201105].

[16] E. Gosset, Y. Nazé, J.-F. Claeskens, G. Rauw, J.-M. Vreux, and H. Sana, An XMM-Newton look at the Wolf-Rayet star WR 40. The star itself, its nebula and its neighbours, A\&A 429 (Jan., 2005) 685-704. 